\title{
A Theorem on Canonical Commutation and Anticommutation Relations
}

\author{
G.-F. Dell'Antonio* \\ Institut des Hautes Etudes Scientifiques \\ S. DOPLICHER \\ Istituto di Fisica dell'Università, Roma \\ and Institut des Hautes Etudes Scientifiques \\ D. RUELLE
}

Institut des Hautes Etudes Scientifiques, Bures-sur-Yvette, (S. et O.) France

Received February 7, 1966

\begin{abstract}
The aim of this note is to characterize representations of the canonical commutation or anticommutation relations which, on a subspace of the "space of test-functions", reduce to a sum of copies of the Fock representation.
\end{abstract}

\section{Generalities ${ }^{1}$}

Let $\mathscr{L}$ be a real separated prehilbert space. We assume that $\mathscr{L}$ is separable. One may in a standard way construct a complex Hilbert space $\mathscr{H}$ (Fock space) and, for each $f \in \mathscr{L}$, operators $a(f), a^{*}(f)$ forming the Fock representation of the canonical commutation relations (CCR) or anticommutation relations $(\mathrm{CAR})$ of $\mathscr{L}$.

In the case of the CAR the operators $a(f), a^{*}(f)$ are bounded and the $C^{*}$-algebra $\mathfrak{A}$ associated with the Fock representation of the CAR is defined as the uniform closure of the algebra generated by all operators $a(f), a^{*}(f)$. In the case of the CCR the operators $\varphi(f)=\frac{1}{\sqrt{2}}\left(a(f)+a^{*}(f)\right)$ and $\pi(f)=\frac{1}{i \sqrt{2}}\left(a(f)-a^{*}(f)\right)$ are self-adjoint and one may define the Weyl operators $U(f)=\exp (i \varphi(f)), V(f)=\exp (i \pi(f))$. The $C^{*}$-algebra $\mathfrak{A}$ associated with the Fock representation of the CCR is defined as the uniform closure of the algebra generated by all operators $U(f), V(f) \cdot \mathfrak{A}$ is irreducible and contains the identity operator 1 of $\mathscr{H}$.

A (CCR or CAR) representation of $\mathscr{L}$ in a complex Hilbert space $\mathfrak{G}$ is defined by a *-homomorphism $\gamma$ of $\mathfrak{A}$ into the bounded operators on $\mathfrak{G}$ such that $\gamma(\mathbf{1})$ is the identity on $\mathfrak{S}$ and, in the case of the CCR the

* Present address: Courant Institute of Mathematical Science, N. Y. U.

1 For a general description of CCR and CAR see GARDING and WIgHTMAN [4]; for CCR see LEW [5] and references given there to earlier work, in particular by Segal; for $C^{*}$-algebras see Dixmier [3]. 
functions $t \rightarrow \gamma(\exp (i t \varphi(f))), t \rightarrow \gamma(\exp (i t \pi(f)))$ of the real variable $t$ are strongly continuous for each $f \in \mathscr{L}$. There are then uniquely defined self-adjoint operators $\varphi_{\gamma}(f), \pi_{\gamma}(f)$ on $\mathfrak{G}$ such that

$$
\gamma(U(f))=\exp \left(i \varphi_{\gamma}(f)\right), \quad \gamma(V(f))=\exp \left(i \pi_{\gamma}(f)\right) .
$$

A linear functional $\varrho$ on $\mathfrak{A}$ which is positive $(\geqq 0)$ and normalized $(\varrho(1)=1)$ is called a state on $\mathfrak{A}$. In the case of the CCR we shall always assume that $\varrho$ is regular in the sense that for all $A, B \in \mathfrak{A}$ and $f \in \mathscr{L}$, the functions $\varrho(A U(t f) B)$ and $\varrho(A V(t f) B)$ of the real variable $t$ are continuous. By the Gel'fand-Segal construction one obtains a complex Hilbert space $\mathfrak{G}$, a vector $\Omega \in \mathfrak{G}$ such that $\|\Omega\|=1$ and a $*$-homomorphism $\gamma$ of $\mathscr{A}$ into the bounded operators on $\mathfrak{S}$ satisfying the properties indicated above, $\Omega$ is cyclic with respect to $\gamma(\mathfrak{A})$ and for all $A \in \mathfrak{A}$ one has

$$
\varrho(A)=(\Omega, \gamma(A) \Omega) \text {. }
$$

A state $\varrho$ on $\mathfrak{A}$ defines thus a cyclic representation of $\mathscr{L}$. Conversely a cyclic representation of $\mathscr{L}$ in $\mathfrak{G}$, defined by a *-homomorphism $\gamma$ of $\mathfrak{A}$ and a normalized vector $\Omega$ cyclic with respect to $\gamma(\mathfrak{A})$, yields a state $\varrho$ by (2), and $\varrho$ determines the representation within unitary equivalence (uniqueness of the Gel'fand construction). Although $\mathscr{L}$ is assumed to be separable $\mathfrak{G}$ will in general not be separable ( $\mathfrak{A}$ is in general not normseparable in the case of the CCR).

If $\Phi \in \mathscr{H}$ and $\|\Phi\|=1$ the state $\omega_{\Phi}$ on $\mathfrak{A}$ defined by

$$
\omega_{\Phi}(A)=(\Phi, A \Phi) \text { for all } A \in \mathfrak{A}
$$

is called a vector state. The Gel'fand representation constructed from $\omega_{\Phi}$ is again the Fock representation.

If $\sigma$ is a density matrix (i.e. a positive ( $\geqq 0$ ) operator with trace 1 ) on $\mathscr{H}$, the state $\varrho_{\sigma}$ on $\mathfrak{A}$ defined by

$$
\varrho_{\sigma}(A)=\operatorname{Tr}(\sigma A) \text { for all } A \in \mathcal{A}
$$

is called a normal state (with respect to the Fock representation).

Let a (CCR or CAR) representation of $\mathscr{L}$ be defined on a Hilbert space $\mathfrak{S}$ by a *-homomorphism $\delta$ of $\mathfrak{A}$ into the bounded operators on $\mathfrak{H}$. If we can write $\mathfrak{G}$ as the completed tensor product

$$
\mathfrak{G}=\mathfrak{G}_{1} \bar{\otimes} \mathfrak{G}_{2}
$$

of two Hilbert spaces $\mathfrak{G}_{1}$ and $\mathfrak{G}_{2}$ in such a way that

$$
\delta=\delta_{1} \otimes \mathbf{1}_{\mathfrak{g}_{2}}
$$

and if the representation defined by $\delta_{1}$ on $\mathfrak{G}_{1}$ is the Fock representation (i.e. if $\delta_{1}$ is implemented by an isometry of $\mathscr{H}$ onto $\mathfrak{G}_{1}$ ) our original representation will be called normal. By choosing an orthonormal basis in $\mathfrak{G}_{2}$ one sees that a normal representation is the same thing as a direct sum of copies of the Fock representation. 
Lemma 1. The Gel'fand representation constructed from a normal state $\varrho_{\sigma}$ on $\mathfrak{A}$ is normal.

Using the spectral decomposition of $\sigma$ we may write for all $A \in \mathfrak{A}$

$$
\varrho_{\sigma}(A)=\sum_{n} c_{n} \omega_{\Psi_{n}}(A)
$$

where the $\Psi_{n}$ are orthonormal vectors of $\mathscr{H}$. Let the normalized vector $\Psi \in \mathscr{H} \bar{\otimes} \mathscr{H}$ be defined by

$$
\Psi=\sum_{n} c_{n}^{\frac{1}{2}} \Psi_{n} \otimes \Psi_{n}
$$

and let the $*$-homomorphism $\delta$ of $\mathfrak{A}$ into the bounded operators of $\mathscr{H} \bar{\otimes} \mathscr{H}$ be defined by

We have then for all $A \in \mathfrak{A}$

$$
\delta(A)=A \otimes \mathbf{1} .
$$

$$
\varrho_{\sigma}(A)=(\Psi, \delta(A) \Psi) \text {. }
$$

Let $\mathfrak{S}_{\Psi}$ be the closure of $\delta(\mathfrak{A}) \Psi$ in $\mathscr{H} \bar{\otimes} \mathscr{H}$. The projection $E$ on $\mathfrak{S}_{\Psi}$ commutes with $\delta(\mathfrak{A})=\mathfrak{A} \otimes 1$ and, since $\mathfrak{A}$ is irreducible, it is of the form $E=1 \otimes E_{0}$ where $E_{0}$ is a projection in $\mathscr{H}$. By the uniqueness of the Gel'fand construction, the Gel'fand representation constructed from $\varrho_{\sigma}$ is defined by the restriction $\delta_{0}$ of $\delta$ to $\mathfrak{S}_{\Psi}$. Lemma 1 follows then from the definition of a normal representation and the relations

$$
\begin{gathered}
\mathfrak{S}_{\Psi}=\mathscr{H} \bar{\otimes} E_{0} \mathscr{H} \\
\delta_{0}(A)=A \otimes \mathbf{1}_{0} \text { for all } A \in \mathfrak{A}
\end{gathered}
$$

where $\mathbf{1}_{0}$ is the identity in $E_{0} \mathscr{H}$.

\section{Number operators}

Let $\gamma$ be a $*$-homomorphism of $\mathfrak{A}$ into the bounded operators of a complex Hilbert space $\mathfrak{S}$ defining a (CCR or CAR) representation of $\mathscr{L}$. If $f \in \mathscr{L}$ we write

$$
\begin{array}{ll}
b(f)=\frac{1}{\sqrt{2}}\left(\varphi_{\gamma}(f)+i \pi_{\gamma}(f)\right) & (\mathrm{CCR}) \\
b(f)=\gamma(a(f)) & (\mathrm{CAR})
\end{array}
$$

where $\varphi_{\gamma}, \pi_{\gamma}$ are given by (1). If $\|f\|=1$ a number operator $N(f)$ $=b(f)^{*} b(f)$ is defined on $\mathfrak{H}, N(f)$ is self-adjoint with spectrum constituted by the non-negative integers (CCR) or 0 and 1 (CAR). We note $E_{n}(f)$ the projection on the subspace corresponding to the eigenvalue $n$ of $N(f)$ so that

$$
N(f)=\sum_{n} n E_{n}(f), \quad \mathbf{1}=\sum_{n} E_{n}(f)
$$

Let $\left(f_{m}\right)$ be an orthonormal basis of $\mathscr{L}$. If $\mathbf{n}=\left(n_{m}\right)$ is a family of nonnegative integers $(\mathrm{CCR})$ or elements of $\{0,1\}$ (CAR) such that $|\mathbf{n}|$ 
$=\sum_{m} n_{m}<+\infty \quad$ we define

$$
\begin{aligned}
& E_{\mathbf{n}}=\prod_{m} E_{n_{m}}\left(f_{m}\right) \\
& E_{n}=\sum_{|\mathbf{n}|=n} E_{\mathbf{n}} .
\end{aligned}
$$

If the ranges of the orthogonal projections $E_{n}$ span $\mathfrak{G}$ we define a selfadjoint operator

$$
N=\sum_{n} n E_{n}
$$

and we have in the sense of strong convergence on the domain of $N$ :

$$
N=\sum_{m} N\left(f_{m}\right) \text {. }
$$

We shall say that the representation has a total number operator $N$ if

1. In the case of the CAR, $N$ exists for one choice of the orthonormal basis $\left(f_{m}\right)$

2. In the case of the CCR, $N$ exists and is the same for every choice ${ }^{2}$ of the orthonormal basis $\left(f_{m}\right)$.

Otherwise we shall say that there is no total number operator.

Lemma $2{ }^{3}$ A representation of $\mathscr{L}$ on $\mathfrak{G}$ is normal if and only if it has a total number operator $N$.

A total number operator is defined for the Fock representation and therefore also for a normal representation.

To prove the converse we first show that, if $N$ exists for one orthonormal basis $\left(f_{m}\right)$ of $\mathscr{L}$, the restriction of the representation to the subspace $\mathscr{L}_{0}$ of $\mathscr{L}$ generated by finite linear combinations of the $f_{m}$ is normal. We assume thus that the ranges $\mathfrak{G}_{\mathbf{n}}$ of the projections $E_{\mathbf{n}}$ span $\mathfrak{G}$.

Let $\mathbf{n}, \mathbf{n}^{\prime}$ be such that $n_{m}-n_{m}^{\prime}=\delta_{m m_{0}}$, then the CCR or CAR show that $b\left(f_{m_{0}}\right) \mathfrak{G}_{\mathbf{n}} \subset \mathfrak{G}_{\mathbf{n}^{\prime}}, b\left(f_{m_{0}}\right) * \mathfrak{G}_{\mathbf{n}^{\prime}} \subset \mathfrak{G}_{\mathbf{n}}$. In fact $\subset$ may be replaced by $=$ in these relations because $n_{m}^{-1} b\left(f_{m_{0}}\right)^{*} b\left(f_{m_{0}}\right)$ reduces to the identity on $\mathfrak{G}_{\mathbf{n}}$ and $n_{m}^{-1} b\left(f_{m_{0}}\right) b\left(f_{m_{0}}\right)^{*}$ reduces to the identity on $\mathfrak{G}_{\mathbf{n}^{\prime}}$. In particular, every vector in $\mathfrak{G}_{\mathbf{n}}$ is of the form $M \Psi$ where $M$ is a monomial in the $b\left(f_{m}\right)^{*}$ and $\Psi \in \mathfrak{S}_{0}$.

Let $\left(\Psi_{\alpha}\right)$ be an orthonormal basis of $\mathfrak{G}_{0}$ and let $\mathfrak{G}_{\alpha}$ be the subspace of $\mathfrak{S}$ spanned by vectors of the form $P \Psi_{\alpha}$ where $P$ is a polynomial in the $b\left(f_{m}\right)^{*}$. The spaces $\mathfrak{S}_{\alpha}$ are orthogonal and the above remarks show that they span $\mathfrak{S}$.

By reference to a standard construction of the Fock space and Fock representation one sees that the representation of $\mathscr{L}_{0}$ on $\mathfrak{g}$ defined by $\gamma$ reduces to the Fock representation on each $\mathfrak{G}_{\alpha}$, it is thus a sum of copies of the Fock representation, i.e. normal.

2 The authors are thankful to I. Segal for pointing out that Lemma 2 is false if one only assumes the existence of $N$ for one basis, counterexamples have been constructed by J. Chaiken (private communication).

${ }^{3}$ See Garding and Wrghtman [4] and Wightman and Schweber [7]. 
In the case of the CAR, the fact that the representation is normal when restricted to $\mathscr{L}_{0}$ implies that it is normal because $\mathscr{L}_{0}$ is dense in $\mathscr{L}$ and $f \rightarrow a(f)$ is continuous (see next footnote).

In the case of the CCR, our assumptions imply that for any $f \in \mathscr{L}$, $a(f)^{*} a(f)$ vanishes on $\mathfrak{G}_{0}$, hence $a(f) \Psi_{\alpha}=0$ for all $\alpha$. Using the commutation relations shows then that the matrix elements of $a(f)$ are those of the Fock representation.

Remark 1. Since the construction of the Fock representation of $\mathscr{L}$ is independent of the choice of a basis in $\mathscr{L}$, Lemma 2 shows that the existence and definition of a total number operator for the CAR are also independent of the choice of a basis.

Remark 2. For a representation of the CAR without total number operator, let $\mathfrak{H}^{\prime}$ be the subspace of $\mathfrak{G}$ spanned by the ranges $\mathfrak{G}_{\mathbf{n}}$ of the projections $E_{\mathbf{n}}$. The above proof shows that the representation leaves $\mathfrak{G}^{\prime}$ stable and that its restriction to $\mathfrak{H}^{\prime}$ is normal.

\section{Normalcy of an induced representation}

Let $\mathscr{L}=\mathscr{L}_{1} \oplus \mathscr{L}_{2}$ where $\mathscr{L}_{1}$ is a real Hilbert space and $\mathscr{L}_{2}$ a real prehilbert space ${ }^{4}$. We let $\mathfrak{A}_{1}, \mathfrak{A}_{2}$ be the $C^{*}$-algebras associated with the Fock representations of $\mathscr{L}_{1}$ resp. $\mathscr{L}_{2}$ in the Fock spaces $\mathscr{H}_{1}$ resp. $\mathscr{H}_{2}$ and $\widetilde{\mathfrak{A}}_{1}, \widetilde{\mathfrak{A}}_{2}$ be the $C^{*}$-subalgebras of $\mathscr{A}$ generated by the $U(f), V(f)$ (CCR) or the $a(f), a^{*}(f)$ (CAR) with $f \in \mathscr{L}_{1}$ resp. $f \in \mathscr{L}_{2}$. One can identify naturally the Fock space $\mathscr{H}$ of $\mathscr{L}$ with the completed tensor product of $\mathscr{H}_{1}$ and $\mathscr{H}_{2}$ :

$$
\mathscr{H}=\mathscr{H}_{1} \bar{\otimes} \mathscr{H}_{2}
$$

in such a way that $\mathfrak{A}_{1} \otimes \mathbf{1}_{2}$ is identified with $\widetilde{\mathfrak{A}}_{1}$. In the case of the CCR, $\mathbf{1}_{1} \otimes \mathfrak{A}_{2}$ is also identified with $\widetilde{\mathfrak{A}}_{2}$ (but this is not so for the CAR since $\widetilde{\mathfrak{A}}_{1}, \widetilde{\mathfrak{A}}_{2}$ do not commute). Notice that it follows from our definitions that the finite sums $\sum_{i} A_{1}^{i} A_{2}^{i}$ with $A_{1}^{i} \in \widetilde{\mathfrak{A}}_{1}, A_{2}^{i} \in \widetilde{\mathfrak{A}}_{2}$ are uniformly dense in $\mathfrak{A}$.

Let a representation of $\mathscr{L}$ be defined by a $*$-homomorphism $\gamma$ of $\mathfrak{A}$ into the bounded operators in a complex Hilbert space $\mathfrak{S}$, and let $\gamma^{\prime}$ be the $*$-homomorphism of $\mathfrak{A}_{1}$ defined by $\gamma^{\prime}(A)=\gamma\left(A \otimes \mathbf{1}_{2}\right)$. We say that the representation of $\mathscr{L}_{1}$ on $\mathfrak{S}$ defined by $\gamma^{\prime}$ is the representation induced by the above one on $\mathscr{L}_{1}$.

We consider now to the case where the representation defined by $\gamma$ is the Gel'fand representation constructed from a state $\varrho$ on $\mathfrak{A}$ (in the case of the CCR we assume as usual that $\varrho$ is regular). There exists then

${ }^{4}$ In the case of the CAR, we might without loss of generality assume that $\mathscr{L}_{2}$ (and therefore $\mathscr{L}$ ) is complete (cf. [2]). We have indeed by virtue of the CAR, $\|a(f)\|=\left\|a^{*}(f)\right\|=\|f\|$; hence $a(f)$ and $a^{*}(f)$ are continuous in $f$ and the $C^{*}$-algebra $\mathfrak{A}_{2}$ generated by the $a(f), a^{*}(f)$ with $f \in \mathscr{L}_{2}$ is identical to the $C^{*}$-algebra generated by the $a(f), a^{*}(f)$ with $f$ in the completion $\overline{\mathscr{L}}_{2}$ of $\mathscr{L}_{2}$. 
a normalized vector $\Omega \in \mathfrak{G}$, cyclic with respect to $\gamma(\mathfrak{Q})$ and such that

$$
\varrho(A)=\omega_{\Omega}(\gamma(A))=(\Omega, \gamma(A) \Omega)
$$

for all $A \in \mathfrak{A}$. With these notations we prove

Theorem. The condition

A. If the projections $E_{n}^{\prime}$ are defined by (16) for the representation defined by the *-homomorphism $\gamma^{\prime}$, then

$$
\sum_{n} \omega_{\Omega}\left(E_{n}^{\prime}\right)=\mathbf{1}
$$

is implied by and, in the case of the $\mathrm{CAR}$, equivalent to, the following equivalent conditions

B. The representation of $\mathscr{L}_{1}$ on $\mathfrak{S}$ defined by $\gamma^{\prime}$ has a total number operator $N^{\prime}$.

C. The representation of $\mathscr{L}_{1}$ on $\mathfrak{G}$ defined by $\gamma^{\prime}$ is normal.

D. There exists a density matrix $\sigma$ on $\mathscr{H}_{1}$ such that

$$
\varrho\left(A \otimes \mathbf{1}_{\mathbf{2}}\right)=\omega_{\Omega}\left(\gamma^{\prime}(A)\right)=\operatorname{Tr}(\sigma A)=\varrho_{\sigma}(A)
$$

for all $A \in \mathfrak{A}_{1}$.

The implication $B \Rightarrow A$ is obvious, and the equivalence $B \Leftrightarrow C$ follows directly from Lemma 2 . We prove now successively $A \Rightarrow B$ (for CAR), $C \Rightarrow D, D \Rightarrow B$.

$A \Rightarrow B(\mathrm{CAR})$

Equation (21) expresses that $\Omega$ is contained in the subspace $\mathfrak{G}^{\prime}$ of $\mathfrak{G}$ spanned by the ranges $\mathfrak{S}_{n}^{\prime}$ of the projections $E_{n}^{\prime}$. We have to prove that $\mathfrak{H}^{\prime}=\mathfrak{G}$, or equivalently that $\gamma(\mathfrak{A}) \Omega\left(\mathfrak{G}^{\prime}\right.$ or, since the finite sums $\sum_{i} A_{1}^{i} A_{2}^{i}$ with $A_{1}^{i} \in \widetilde{\mathfrak{Q}}_{1}, A_{2}^{i} \in \widetilde{\mathfrak{A}}_{2}$ are uniformly dense in $\mathfrak{A}$, that $\gamma\left(\widetilde{\mathfrak{A}}_{1}\right) \times$ $\times \gamma\left(\widetilde{\mathfrak{A}}_{2}\right) \Omega \subset \mathfrak{S}^{\prime}$. Since $\Omega \in \mathfrak{G}^{\prime}$ and $\gamma\left(\widetilde{\mathfrak{Q}}_{2}\right) \mathfrak{S}_{n}^{\prime} \subset \mathfrak{G}_{n}^{\prime}$ it remains to check that $\gamma\left(\widetilde{\mathfrak{A}}_{1}\right) \mathfrak{H}^{\prime}=\gamma\left(\mathfrak{A}_{1} \otimes \mathbf{1}_{2}\right) \mathfrak{G}^{\prime}=\gamma^{\prime}\left(\mathfrak{A}_{1}\right) \mathfrak{G}^{\prime} \subset \mathfrak{G}^{\prime}$, but this follows from Remark 2 at the end of Section 2.

$$
C \Rightarrow D
$$

By definition, condition $B$ means that one can write $\mathfrak{S}=\mathfrak{G}_{1} \bar{\otimes} \mathfrak{G}_{2}$ and $\gamma^{\prime}=\gamma_{1}^{\prime} \otimes \mathbf{1}_{\mathfrak{f}_{2}}$ where $\gamma_{1}^{\prime}$ is implemented by an isometry $W$ of $\mathscr{H}_{1}$ onto $\mathfrak{G}_{1}$. Let $\left(\Psi_{\alpha}\right)$ be an orthonormal basis of $\mathfrak{G}_{2}$, then an isometry $W_{\alpha}$ of $\mathscr{H}_{1}$ onto $\mathfrak{G}_{1} \otimes \Psi_{\alpha}$ is defined by $W_{\alpha} \Psi=W \Psi \otimes \Psi_{\alpha}$. Let $c_{\alpha}^{1 / 2} \Omega_{\alpha}$ be the component of $\Omega$ in $\mathfrak{G}_{1} \otimes \Psi_{\alpha}$, where $\left\|\Omega_{\alpha}\right\|=1$; we have then

and for all $A \in \mathfrak{R}_{1}$

$$
\sum_{\alpha} c_{\alpha}=1
$$

$$
\begin{aligned}
\varrho\left(A \otimes \mathbf{1}_{\mathbf{2}}\right) & =\omega_{\Omega}\left(\gamma^{\prime}(A)\right)=\sum_{\alpha} c_{\alpha} \omega_{\Omega_{\alpha}}\left(W_{\alpha} A W_{\alpha}^{-1}\right) \\
& =\sum_{*} c_{\alpha}\left(W_{\alpha}^{-1} \Omega, A W_{\alpha}^{-1} \Omega_{\alpha}\right) .
\end{aligned}
$$


If $E_{\alpha}$ is the projection on $W_{\alpha}^{-1} \Omega_{\alpha}$ in $\mathscr{H}_{1},(23)$ implies that

$$
\sigma=\sum_{\alpha} c_{\alpha} E_{\alpha}
$$

is a density matrix and $D$ follows from (24) and (25).

$D \Rightarrow B$

Let $\mathfrak{S}_{\Omega}$ be the closure in $\mathfrak{H}$ of $\gamma^{\prime}\left(\mathfrak{R}_{1}\right) \Omega$. By the uniqueness of the Gel'fand construction, the restriction to $\mathfrak{G}_{\Omega}$ of the representation defined by $\gamma^{\prime}$ in $\mathfrak{S}$ is, by (22), identical to the Gel'fand representation constructed from the normal state $\varrho_{\sigma}$ on $\mathfrak{A}_{1}$ and thus normal by Lemma 1 . This restricted representation has thus a total number operator $N^{\prime \prime}$. From this follows the existence of a total number operator $N^{\prime}$ for the representation defined by $\gamma^{\prime}$ in $\mathfrak{G}:$ if $\Psi \in \mathfrak{F}_{\Omega}$ and $A_{2} \in \mathfrak{A}_{2}$, then $N^{\prime} \gamma\left(A_{2}\right) \Psi$ $=\gamma\left(A_{2}\right) N^{\prime \prime} \Psi$.

Remark. In the case of the CCR, $C$ may be rewritten as $C^{\prime}$. One may write $\mathfrak{H}=\mathfrak{H}_{1} \otimes \mathfrak{H}_{2}$ and there exist $*$-homomorphisms $\gamma_{1}^{\prime}, \gamma_{2}^{\prime}$ of $\mathfrak{A}_{1}$, resp. $\mathfrak{A}_{2}$ into the bounded operators on $\mathfrak{G}_{1}$ resp. $\mathfrak{S}_{2}$ such that

$$
\gamma\left(A_{1} \otimes A_{2}\right)=\gamma_{1}^{\prime}\left(A_{1}\right) \otimes \gamma_{2}^{\prime}\left(A_{2}\right)
$$

and $\gamma_{1}^{\prime}$ is implemented by an isometry of $\mathscr{H}_{1}$ onto $\mathfrak{H}_{1}$.

It is clear that $C^{\prime} \Rightarrow C$. To prove that $C \Rightarrow C^{\prime}$ we note that $C$ implies the existence of the decomposition $\mathfrak{H}=\mathfrak{G}_{1} \bar{\otimes} \mathfrak{G}_{2}$ and of $\gamma_{1}^{\prime}$ such that $\gamma^{\prime}=\gamma_{1}^{\prime} \otimes 1_{\mathfrak{S}_{2}}$ and $\gamma_{1}^{\prime}$ is implemented by an isometry of $\mathscr{H}_{1}$ onto $\mathfrak{S}_{1}$. Since $\gamma\left(\mathbf{1}_{1} \otimes \mathfrak{A}_{2}\right)$ is in the commutant of $\gamma\left(\mathfrak{A}_{1} \otimes \mathbf{1}_{2}\right)=\gamma^{\prime}\left(\mathfrak{R}_{1}\right)$ $=\gamma_{1}^{\prime}\left(\mathfrak{Q}_{1}\right) \otimes \mathbf{1}_{\mathfrak{F}_{\mathfrak{z}}}$ and since $\gamma_{1}^{\prime}\left(\mathfrak{H}_{1}\right)$ is irreducible, every operator $\gamma\left(\mathbf{1}_{1} \otimes A_{2}\right)$ with $A_{2} \in \mathfrak{A}_{2}$ is of the form $\mathbf{1}_{1} \otimes \gamma_{2}^{\prime}\left(A_{2}\right)$, which concludes the proof.

\section{Physical interpretation}

The mathematical situation described by the theorem of Section 3 is of interest in the study of quantum mechanical systems with an infinite number of degrees of freedom. For instance, if $\mathscr{L}$ is the space of real square-integrable functions with compact support in $R^{v}, \varrho$ may be taken to be the expectation value functional describing the state of an infinite system of bosons (CCR) or fermions (CAR) in thermodynamic equilibrium in $R^{\nu}$ (see [1], [2] and [6]). Let then $\mathscr{L}_{1}$ be the space of real square-integrable functions on a bounded (measurable) subset $\Lambda$ of $R^{p}$. The restriction of $\varrho$ to $\widetilde{\mathscr{A}}_{1}=\mathfrak{A}_{1} \otimes \mathbf{1}_{\mathbf{2}}$ will describe the particles contained in the region $A$. Condition $\mathrm{B}$ (or $\mathrm{A}$ : in the case of CAR they are equivalent) expresses that the probability of finding an infinite number of particles simultaneously in $\Lambda$ vanishes. This condition is always satisfied for particles with hard cores; in general its violation would correspond to a catastrophic behaviour of the system from the thermodynamic point of view. The theorem tells us then that the restriction of $\varrho$ to the region $A$ (i.e. to $\mathfrak{A}_{1} \otimes \mathbf{1}_{2}$ ) is given by a density matrix. For more details see [6]. 
Acknowledgements. The authors are indebted to Prof. R. KaDison for helpful discussions and comments on an early draft or this work. They are also grateful to I. Segal for pointing out an important error in the original manuscript. Two of us (G.- F. D., S. D.) wish to thank M. L. Motchane for his kind hospitality at the I. H. E. S.

\section{References}

[1] Araki, H., and E. J. Woods: J. Math. Phys. 4, 637 (1963).

[2] -, and W. Wyss: Helv. Phys. Acta 37, 136 (1964).

[3] Dixmier, J.: Les $C^{*}$-algèbres et leurs représentations. Paris: Gauthier-Villars 1964.

[4] Garding, L., and A. Wightman: Proc. Nat. Acad. Sci. 40, 617 (1954); 40, 622 (1954).

[5] Lew, J. S.: Thesis, Princeton 1960 (unpublished).

[6] RUELle, D.: Lecture notes of the summer school of theoretical physics. Cargèse, Corsica. July 1965.

[7] Wightman, A. S., and S. S. Schweber: Phys. Rev. 98, 812 (1955). 